EPJ Web of Conferences 47, 05008 (2013)

DOI: $10.1051 /$ epjconf/20134705008

(C) Owned by the authors, published by EDP Sciences, 2013

\title{
Multiplicity and properties of Kepler planet candidates: High spatial imaging and RV studies*
}

\author{
D. Barrado ${ }^{1,2, a}$, J. Lillo-Box ${ }^{2, b}$, H. Bouy ${ }^{2}$, J. Aceituno ${ }^{1}$ and S. Sánchez ${ }^{3}$ \\ ${ }^{1}$ Centro Astronómico Hispano-Alemán (CAHA). Calar Alto Observatory, c/ Jesús Durbán \\ Remón 2-2, 04004 Almería, Spain \\ ${ }^{2}$ Departamento de Astrofísica, Centro de Astrobiologíia, ESAC campus 28691 Villanueva de \\ la Cañada (Madrid), Spain \\ ${ }^{3}$ Instituto de Astrofísica de Andalucía (IAA/CSIC), Glorieta de la Astronomía s/n Aptdo. 3004, \\ 18080 Granada, Spain
}

\begin{abstract}
The Kepler space telescope is discovering thousands of new planet candidates. However, a follow up program is needed in order to reject false candidates and to fully characterize the bona-fide exoplanets. Our main aims are: 1./ Detect and analyze close companions inside the typical Kepler PSF to study if they are the responsible of the dim in the Kepler light curves, 2./ Study the change in the stellar and planetary parameters due to the presence of an unresolved object, 3./ Help to validate those Kepler Objects of Interest that do not present any object inside the Kepler PSF and 4./ Study the multiplicity rate in planet host candidates. Such a large sample of observed planet host candidates allows us to do statistics about the presence of close (visual or bounded) companions to the harboring star. We present here Lucky Imaging observations for a total amount of 98 Kepler Objects of Interest. This technique is based on the acquisition of thousands of very short exposure time images. Then, a selection and combination of a small amount of the best quality frames provides a high resolution image with objects having a 0.1 arcsec PSF. We applied this technique to carry out observations in the Sloan $i$ and Sloan $z$ filters of our Kepler candidates. We find blended objects inside the Kepler PSF for a significant percentage of KOIs. On one hand, only $58.2 \%$ of the hosts do not present any object within 6 arcsec. On the other hand, we have found 19 companions closer than 3 arcsec in 17 KOIs. According to their magnitudes and $i-z$ color, 8 of them could be physically bounded to the host star. We are also collecting high-spectral resolution spectroscopuy in order to derive the planet properties.
\end{abstract}

\section{INTRODUCTION}

It is not so long when the discovery of extrasolar planets was just utopian. However, after the first discovery of an exoplanet orbiting a main sequence star [1,2], the scientific community has discovered and confirmed hundreds of these objects around other stars or even in isolation ([3], [4]). In this context, the Kepler Space Telescope has detected a new large sample of planet host candidates by continuously observing more than 150000 stars in a specific field of view $\left(\mathrm{RA}=19 \mathrm{~h} 22 \mathrm{~m} 40 \mathrm{~s}\right.$ DEC $\left.=+4430^{\prime} 00^{\prime \prime}\right)$. During the first five quarters of observations (i.e, $\approx 4.5$ months) the Kepler Team collected on its second public release a total amount of 997 planet host star candidates [second public release, 5].

\footnotetext{
ae-mail: barrado@cab.inta-csic.es

be-mail: jlillo@cab.inta-csic.es
}

*Based on observations collected at the German-Spanish Astronomical Center, Calar Alto, jointly operated by the Max-PlanckInstitut für Astronomie Heidelberg and the Instituto de Astrofísica de Andalucía (CSIC)

This is an Open Access article distributed under the terms of the Creative Commons Attribution License 2.0, which permits unrestricted use, distribution, and reproduction in any medium, provided the original work is properly cited. 


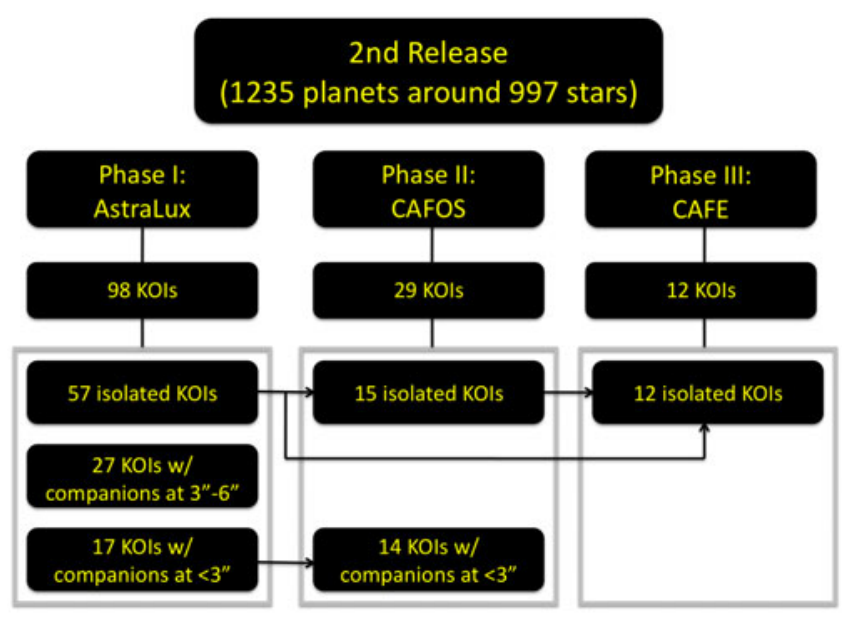

Figure 1. A three phases study: from high spatial resolution imaging, to reject false posives and study multiplicity of the host star, to high-spectral resolution for RV (and planet properties). Additional informaction can be found in [8], [9] and [10].

In this paper, we present a comprehensive study of Kepler candidates. A set of high resolution images obtained with the Lucky-Imaging technique [6] in the $2.2 \mathrm{~m}$ telescope in Calar Alto Observatory (Almería, Spain) with the AstraLux instrument were acquired. This technique allows us to obtain diffraction limited observations with the best seeing conditions in the SDSS $i$ band (see [7] and his $\mathrm{PhD}$ dissertation). A total amount of 98 KOIs (i.e., about a $10 \%$ of the candidates listed in the Kepler second public release of the Kepler Team) have been pointed and studied.

\section{PLANNING, OBSERVATIONS AND ANALYSIS}

\subsection{Planning}

Our project consists in three distinct phases. An scheme can be found in Fig. 1, where we also detail the instruments we have used at the Calar Alto observatory. The goal of the first is to reject false positive due to different possible configurations (eclipsing binaries -EB- and other effects, see Fig. 2).

Phase I) Astralux imaging.- To obtain deep imaging ( $\left.i_{\text {lim }} \approx 23 \mathrm{mag}\right)$ with high spacial resolution $(\approx 0 " .1)$ to reject false positives and to derive colors for the faint companions. See [8]).

Phase II) CAFOS spectroscopy.- For those planet candidates passing the previous test, low- and medium-resolution spectroscopy, in order to reject false positives (by putting limits on the RV), perform spectral typing and study the properties of the host star: metallicity, chromospheric emission and youth. See [9]).

Phase III) CAFE.- For those passing phase II, we have used the new echelle spectrograph in order to obtain radial velocities and derive masses for the star and the planet. See [10]).

\subsection{Sample selection and observations}

We have high spatial resolution for 98 KOIs. The majority of them were selected due to the lack of any kind of follow-up observations according to the published data of the Kepler Team [5, second public release]. The selection of the rest was coordinated with the Kepler Team. In addition, we limit our targets to be brighter than 15 magnitudes in the Sloan $i$ band in order to detect objects five magnitudes 


\section{Hot Planets and Cool Stars}

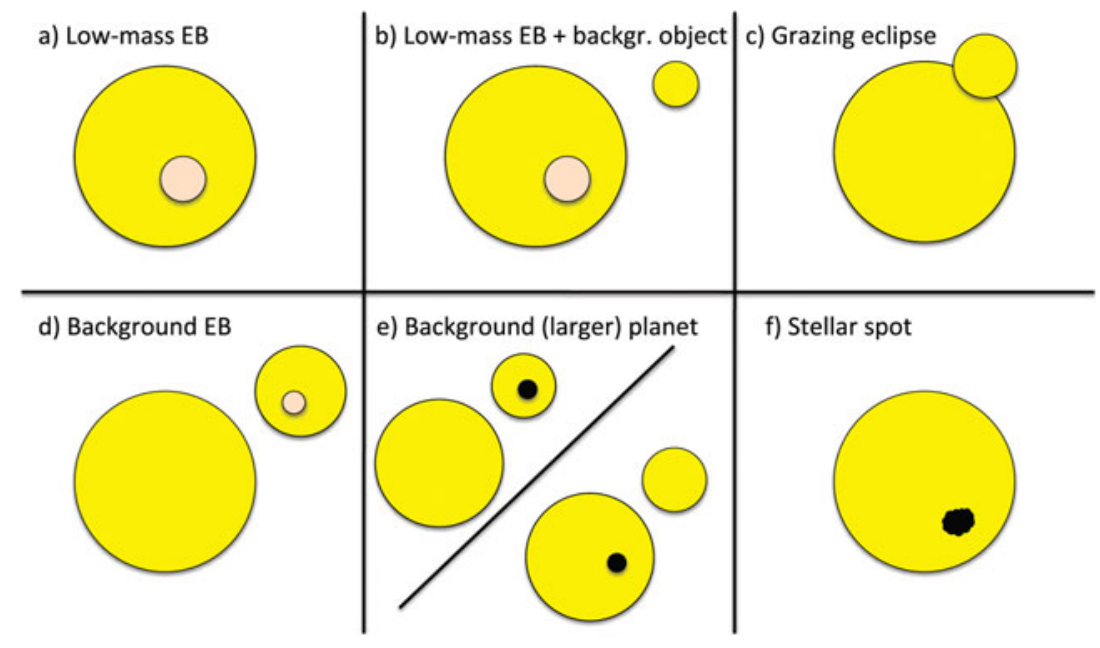

Figure 2. Possible false positive configurations for the Kepler planet candidates. In all panels, the largest circle represents the host star, the orange circle represents a red low-mass star and the black dot represent a planet. A star with a stellar spot is displayed in panel $f$ ). Configuration $e$ ) is not a real false positive, but the properties of the planets require a re-evaluation.

fainter than the KOI. Apart from these constrains, KOIs were randomly selected from the remaining sample so that we expect no bias in our results.

The data presented in this paper was taken in the Calar Alto Observatory (Almería, Spain) within 19 nights of observations divided in three separate runs (06-12 June 2011, 01-10 July 2011 and 25-26 July 2011). Although two nights were lost due to weather conditions, the remaining 17 had very good atmospheric stability with low atmospheric extinction and sporadic or absent clouds, ideal for this observing technique. The mean natural seeing was around 0.9 arcsec over the entire run.

We used the AstraLux instrument placed at the 2.2 meter telescope to perform diffraction limited imaging of our sample of KOIs with the Lucky Imaging technique. We used the entire available field of view of the camera (i.e., $24 \times 24 \mathrm{arcsec}$ ) to cover a separation range encompassing the entire mean Kepler PSF (6-10 arcsec, siee Fig. 3). In brief, we took thousands of images with short exposure times (100-200 milliseconds), well below the typical atmospheric turbulence changes (see [7] and his $\mathrm{PhD}$ dissertation). We set the exposure times and number of frames according to the atmospheric conditions and target magnitude in order to reach at least five magnitudes fainter than the central KOI. Thus, our total exposure times are in the range 340-2000 seconds. We note that fainter objects than this magnitude difference would not affect the planet-star properties by more than $0.5 \%$ in the case of the $R_{p} / R_{*}$ rate, see equation (6) in [11]. Moreover the probability that Kepler observations have detected a transit with a signal to noise ratio greater than 7.1 decreases as the star gets fainter. Thus, we designed our observations to reach $\Delta m_{i} \approx 5.0$ mag at $1.0-1.5 \operatorname{arcsec}$ from the KOI.

Images were firstly obtained in the SDSS $i$ filter since the PSF deformation is lower for this band than for shorter wavelengths (this result is quite similar for the SDSS $z$ band). An on-the-fly reduction allow us to check inmediately for close sources. If any, SDSS $z$ band images were acquired in order to characterize the secondary object by using the $i-z$ color.

Additional information regarding the reduction and calibration can be found in [8].

\subsection{Results regarding multiplicity}

One key point to provide a context to our results is the sensitivity of our Lucky imaging. Sensitivity functions were compiled for each image to determine the depth in angular separation and magnitude (actually, $\Delta m$ ) to which we are complete for $3-\sigma$ detection threshold. 


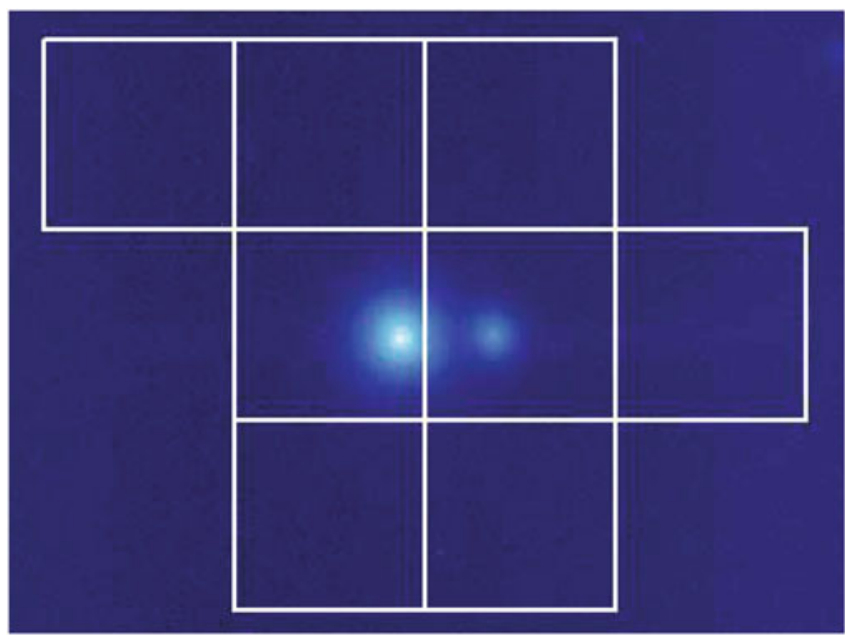

Figure 3. Our Astralux image with the Kepler psf overimposed. Each kepler pixel is $4 \times 4 \operatorname{arcsec}$ large and several pixels are used in the aperture. As in this image, several objects could lie inside the aperture, affecting photometry and eventually making planet smaller than they are.

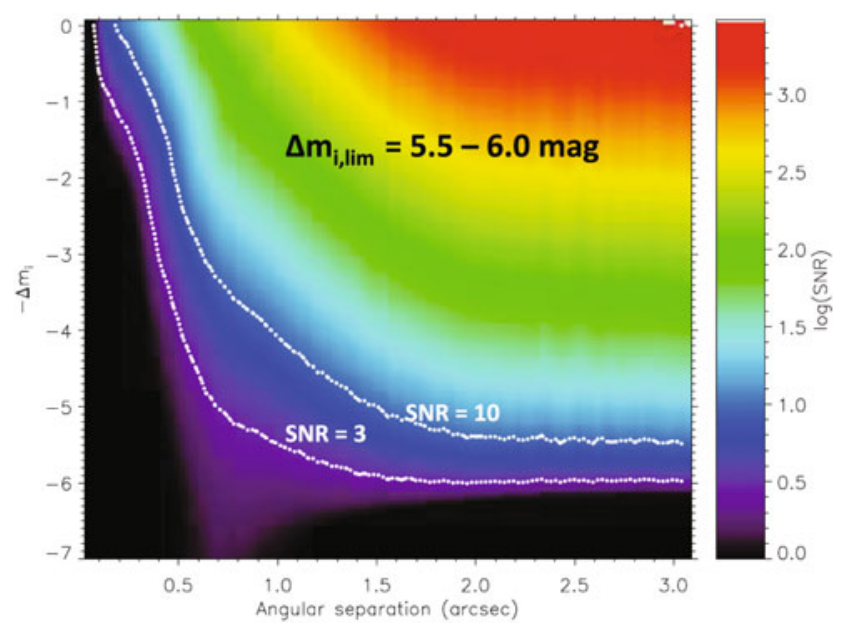

Figure 4. Our sensitivity diagrams indicate that we reach diferences in the range 5.5-6-0 mag for SNR $=3$ detections within one arcsec from the central KIO and 3.0 mag for objects located at 0.4 arcsec.

By measuring the observed radial profile averaged over a large number of directions (avoiding those angles in the way to the close companions) we can infer the dependence of the primary star brightness along the angular separation. Then, we reiteratively add the same profile but located at different angular separations between $0.2-3.0$ arcsec and scale it to be $\Delta m$ magnitudes fainter according to the expression: $F_{/ C}=F_{P} 10^{-0.4 \Delta m}$, being $F_{/ C}$ the encircled flux of the companion and $F_{P}$ the flux of the primary. We then measure the signal to noise ratio (SNR) for the detection of the added profile. Figure 4 shows an example of these calculations where the artificial companion to the observed KOI profile range differential magnitudes of $\Delta m=[0,7]$ and angular separations between 0.2-3 arcsec.

We have found 111 companion sources at angular separations between 0.3-10 arcsec from the 98 targeted KOIs. Since the Kepler point spread function varies from 6 to 10 arcseconds, we consider a KOI 


\section{Hot Planets and Cool Stars}
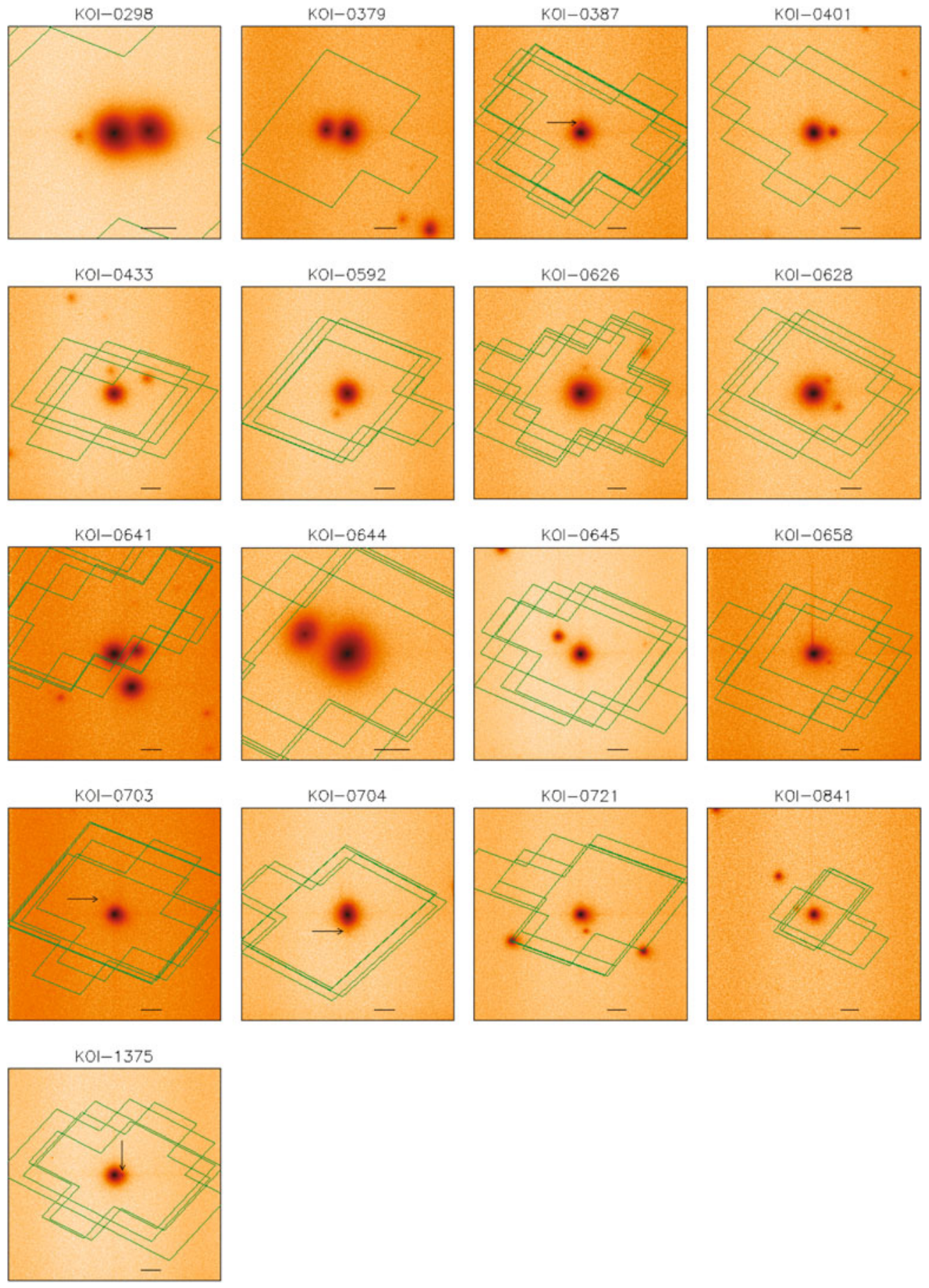

Figure 5. High resolution SDSSi images of the close companions detected around 3 arcsec of 17 Kepler Objects of Interest. North towards up and East towards left. Different sizes are used for each image to clearly show all features. Black arrows indicate the position of the faintest targets and the horizontal black line represents 2 arcsec on each image. Green polygons represent the optimal public Kepler apertures used on each Quarter of the Kepler Mission to compute the light curves. The brighter object assumed to be the $\mathrm{KOI}$ is centered in the images for reconnaissance purposes. 

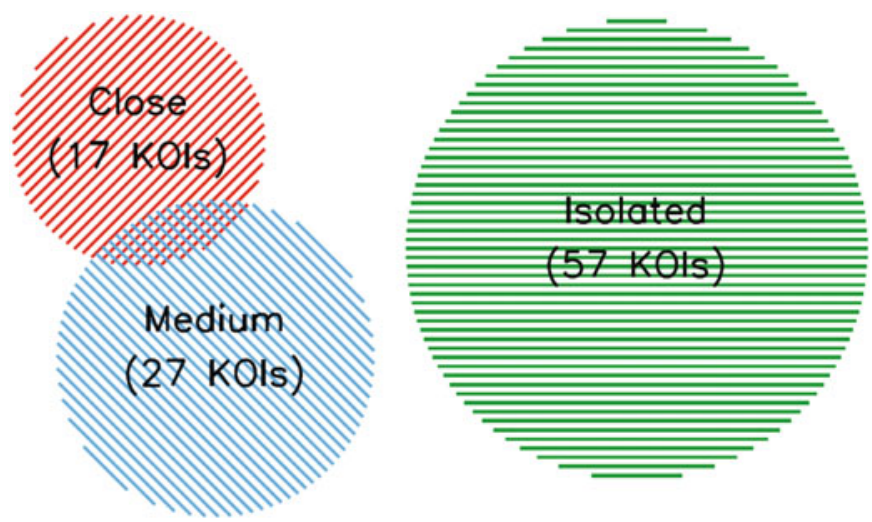

Figure 6. Main multiplicity results of our survey. Isolated KOIs are represented by the green color. KOIs with objects between 3-6 arcsec are represented by the blue color. The close companion group (KOIs with at least one source between 0.3-3.0 arcsec) is shown in red. Note that three KOIs (KOI-0433, KOI-0641 and KOI-0841) have objects either in the close and medium distance groups.

to be isolated if no objects below 6 arcseconds are found in our Lucky Imaging observations. Objects with larger angular separations could be easily detected by the Kepler images or previous ground-based all sky surveys.

According to this definition, we have detected 57 isolated KOIs. This means a $58.2 \%$ rate of isolated objects for planet host candidates in the Kepler Objects of Interest catalog. It is important to remark that this isolation rate would decrease down to the $33.7 \%$ if we consider a 10 arcsec PSF for Kepler observations. However, the best PSF assumption of 6 arcsec will be considered in this paper.

Regarding KOIs with visible companions inside 6 arcsec, 41 planet host candidates have, at least, one object within this projected separation (i.e., 41.8\%). Among them, 17 KOIs present a total amount of 19 companions at less than $3 \operatorname{arcsec}(17.3 \%$ of all KOIs considered). We will refer to this group along this paper as the close companions' group. In Fig. 5 we show the 17 KOIs with close companions and the Kepler optimal (public) apertures of the different Quarters in which the Kepler Mission is divided. All 19 companions lie inside these apertures contaminating the light curve fluxes with its relevant impact in the planet-star properties and planet validation. Moreover, 27 KOIs do present 30 companions within the range 3-6 arcsec (27.6\% of all KOIs considered), medium distance group. This means that three of the main targets from the close companion group (KOI-0433, KOI-0641 and KOI-0841, see Fig 5) present either close $(<3$ arcsec) and medium-separation $(3-6 \operatorname{arcsec})$ sources. Figure 6 summarizes these results.

\subsection{Updating planet-star parameters}

The incidence in the planet and host star parameters due to the presence of a bounded or visual companion to the host star can be measured by isolating the contribution of the host star from the blended companion. Although the transit depth defined as $F_{\text {notransit }}-F_{\text {transit }}$ do not depends on the presence of blended objects in the PSF, physical properties of the planet-star system actually depend on the quotient $\Delta F=\left(F_{\text {notransit }}-F_{\text {transit }}\right) / F_{\text {notransit }}$ as shown by [11]. This quantity is clearly decreased with the closeness of a blended object since it would contribute with an additional flat flux (an intrinsic variability of the blended star would be visible in the light curve $)$. Hence, a change of $\left(F_{\text {notransit }}-F_{\text {blend }}\right) / F_{\text {notransit }}$ will imply consequent changes in the planet-star parameters. Additional information can be found in [8]. 

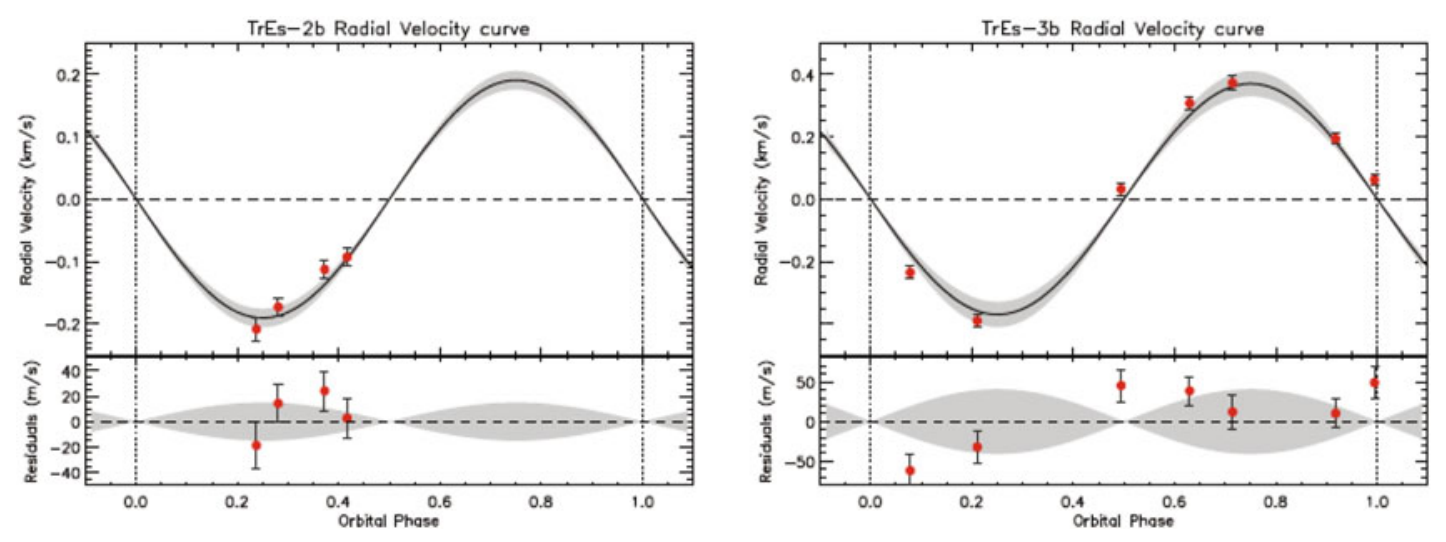

Figure 7. Radial velocity curves for two well-known extra-solar planets, TrEs-2b (left-panel), and TrEs-3b (rightpanel), derived from the early measurements taken during the CAFE commissioning run. Red circles represent the derived values for the radial velocity. The black solid line represents the theoretical curve. The shaded region has been calculated by error propagation of the published values in the previous expression. The lower panel shows the residuals for the fit.

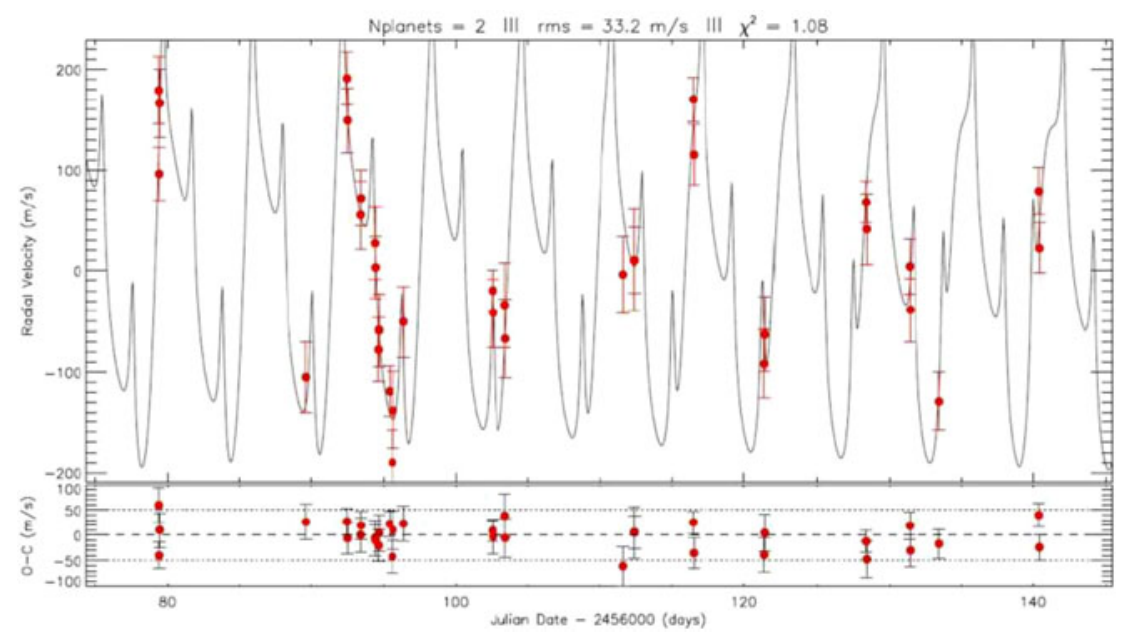

Figure 8. One of our Kepler targets, observed with the CAFE high spectral resolution spectrograph. We have confirmed the presenece of a transiting planet and discovered another via the radial velocity signature. See [10] for details.

\subsection{Radial velocity: Planet properties}

After finishing with the host star characterization (essentially, the high-spatial imaging), we have moved to the planet charcterization by obtaining accurate radial velocities. This is an on-going project and we will only provide an brief overview of the current status.

The observations are being carried out with the new echelle spectrograph at the $2.2 \mathrm{~m}$ telescope at the Calar Alto Observatory (see [12]. During the commissioning data we have proved that the instrument is very stable, can be used for exoplanetary studies (see Fig. 7) and currently the accuracy can reach about $\sim 20 \mathrm{~m} / \mathrm{s}$.

We have already confirmed several Kepler candidates (see Fig. 8), and even identify new planetary systems (ie, those KIOs with more than one planet). Since we are in the process of acquiring data 
(several long orbital periods are among our targets), we defer any additional discussion to a forthcoming paper ([10]). However, we can advance that CAFE is a reliable planet hunter and it has a nice niche: the characterization of systems displaying radial velocity variations of few tens of $\mathrm{m} / \mathrm{s}$.

\section{SUMMARY AND CONCLUSIONS}

We have started a high resolution imaging and spectrocopy follow-up for the Kepler sample of planet host candidates. The main goal of this survey is to provide additional constrains for the confirmation of the planetary nature of these candidates, identify those that are possible false positives and stablish the poperties of the exoplanetary systems. A total amount of 98 KOIs (out of the 997) from the second release of the Kepler Team have been properly observed by using the Lucky Imaging technique with the AstraLux instrument at the $2.2 \mathrm{~m}$ telescope at Calar Alto Observatory. Several dozens are being follow-up with the CAFE echelle spectrograph.

Our main results show that the $58.2 \%$ of the KOIs are actually isolated in terms of not having any visual or bounded companion at less than 6 arcsec. In other words, the $41.9 \%$ of the candidates present close objects. This is an important result in terms of: (a) False positive rate determination, since it points directly which objects have stellar companions that can be mimicking a planet transit detected by Kepler, being then the highest priority for a deeper follow-up with ground-based telescopes to determine the nature of this transit; (b) Updating the planet properties, since they depend on the brightness of the host star. We warn that orbital and physical parameters of the 23 planets orbiting the 17 KOIs with close stellar companions should be revised. (c) Estimating the binary rate in planet host stars. We have shown (see Lillo-Box et al. 2012) that between 6 and 9 of the close companions could be actually bounded to the host star due to their position over our empirical ZAMS. Their distances agree with an S-type binary although still more observations are necessary to confirm both the planet and the binary in all cases. KOIs 0379B, 0658B, 0641B, 0645B and 0298B clearly lie over the ZAMS which suggests a simultaneous formation together with the primary star. Moreover, KOIs 0433B, 0401B, 0592B and 0703B could also lie inside the error bars of the ZAMS but more work should be done to confirm this result. If confirmed, it would imply a lower limit on the observed binary frequency of 6.2-9.2\% Regarding the medium-distance companions (3-6 arcsec), we concluded that only one of them (KOI0623B) is possibly bounded although we should flag this result due to the large errors in the distance estimates.

Subsequent radial velocity observations with the new echelle spectrograph CAFE confirm the planetary nature of some of the isolated targets mentioned above. Some examples can be found in Fig. 7, corresponding to the commissionning data, and Fig. 8, to a KIO, where we ahve confirmed a planets and possible identified another. Additional details will be presented in a forthcoming paper ([10]).

\section{References}

[1] M. Mayor, D. Queloz, Nature 378, 355 (1995)

[2] G.W. Marcy, R.P. Butler, ApJ Letters 464, L147 (1996)

[3] D. Barrado y Navascués, J.R. Stauffer, J. Bouvier, E.L. Martín, ApJ 546, 1006 (2001)

[4] D. Barrado y Navascués, M.R. Zapatero Osorio, E.L. Martín, V.J.S. Béjar, R. Rebolo, R. Mundt, A\&A Letters 393, L85 (2002)

[5] W.J. Borucki, D.G. Koch, G. Basri, N. Batalha, T.M. Brown, S.T. Bryson, D. Caldwell, J. Christensen-Dalsgaard, W.D. Cochran, E. DeVore et al., ApJ 736, 19 (2011)

[6] N.M. Law, C.D. Mackay, J.E. Baldwin, A\&A 446, 739 (2006)

[7] F. Hormuth, W. Brandner, S. Hippler, T. Henning, Journal of Physics Conference Series 131, 012051 (2008)

[8] J. Lillo-Box, D. Barrado, H. Bouy, A\&A 546, A10 (2012) 


\section{Hot Planets and Cool Stars}

[9] J. Lillo-Bos, D. Barrado, In prep (2013)

[10] J. Lillo-Bos, D. Barrado, et al., In prep (2013)

[11] S. Seager, G. Mallén-Ornelas, ApJ 585, 1038 (2003)

[12] J. Aceituno, S.F. Sanchez, F. Grupp, J. Lillo, M. Hernan-Obispo, D.Benitez, L.M. Montoya, U. Thiele, S. Pedraz, D. Barrado et al., ArXiv e-prints (2013) 